\title{
Use of cardiopulmonary exercise testing to assess early ventilatory changes related to occupational particulate matter
}

\author{
T.P. Chao ${ }^{1}$, E.F. Sperandio ${ }^{1}$, T.L.V.P. Ostolin ${ }^{1}$, V.R. Almeida ${ }^{1}$, M. Romiti ${ }^{2}$, A.R.T. Gagliardi ${ }^{2}$, \\ R.L. Arantes ${ }^{2}$ and V.Z. Dourado \\ ${ }^{1}$ Laboratório de Epidemiologia e Movimento Humano, Departamento de Ciências do Movimento Humano, \\ Universidade Federal de São Paulo, Santos, SP, Brasil \\ ${ }^{2}$ Instituto de Medicina Cardiovascular Angiocorpore, Santos, SP, Brasil
}

\begin{abstract}
Spirometry has been used as the main strategy for assessing ventilatory changes related to occupational exposure to particulate matter (OEPM). However, in some cases, as one of its limitations, it may not be sensitive enough to show abnormalities before extensive damage, as seen in restrictive lung diseases. Therefore, we hypothesized that cardiopulmonary exercise testing (CPET) may be better than spirometry to detect early ventilatory impairment caused by OEPM. We selected 135 male workers with at least one year of exposure. After collection of self-reported socioeconomic status, educational level, and cardiovascular risk data, participants underwent spirometry, CPET, body composition assessment (bioelectrical impedance), and triaxial accelerometry (for level of physical activity in daily life). CPET was performed using a ramp protocol on a treadmill. Metabolic, cardiovascular, ventilatory, and submaximal relationships were measured. We compared 52 exposed to 83 non-exposed workers. Multiple linear regressions were developed using spirometry and CPET variables as outcomes and OEPM as the main predictor, and adjusted by the main covariates. Our results showed that OEPM was associated with significant reductions in peak minute ventilation, peak tidal volume, and breathing reserve index. Exposed participants presented shallower slope of $\Delta \mathrm{VT} / \Delta \operatorname{InV} \mathrm{E}$ (breathing pattern), i.e., increased tachypneic breathing pattern. The OEPM explained $7.4 \%$ of the $\Delta \mathrm{VT} / \Delta \ln \mathrm{V} E$ variability. We found no significant influence of spirometric indices after multiple linear regressions. We conclude that CPET might be a more sensitive feature of assessing early pulmonary impairment related to OEPM. Our cross-sectional results suggested that CPET is a promising tool for the screening of asymptomatic male workers.
\end{abstract}

Key words: Occupational exposure to particulate matter; Cardiopulmonary exercise testing; Detection; Spirometry; Ventilatory impairment

\section{Introduction}

Occupational exposure to particulate matter (OEPM) is responsible for the increase in mortality and morbidity rates in workers by cardiorespiratory diseases (1-3). Spirometry has been used as the first-choice instrument for the evaluation of pulmonary alterations in individuals vulnerable to OEPM $(4,5)$. Currently, the instrument is indicated for diagnosing the risk of damage, identifying lung disease, monitoring subjects exposed to harmful particulate matter, as well as to evaluate therapeutic interventions (6). However, in some cases, as one of its limitations, it might not be sensitive enough to show abnormalities before extensive damage as seen in restrictive lung diseases (7).

The evaluation of cardiorespiratory fitness with cardiopulmonary exercise testing (CPET) is used for the diagnosis of exercise intolerance as well as prognosis of patients with chronic diseases (8). Because of the complexity of using exercise testing, imaging information regarding cardiac structure and function as well as ventilatory gas exchange measurements are used to detect small changes that reflect the functional capacity of the cardiovascular, respiratory, and musculoskeletal systems. These measurements respond to different metabolic demands in health and disease conditions (9). Therefore, compared to spirometry, CPET could be a more sensible respiratory test to detect early ventilatory impairment caused by OEPM.

CPET has been explored in the literature regarding its prognostic and diagnostic value in cardiorespiratory diseases, as well as in cardiac and pulmonary rehabilitation. However, there are few studies in the literature 
focusing on the potential of CPET for screening early pulmonary alterations caused by OEPM. It has been shown that CPET is an appropriate tool to assess heterogeneous abnormalities related to respiratory mechanics and pulmonary gas exchange that are not detected early by traditional resting pulmonary function tests, e.g., spirometry. CPET can also explain the incipient symptoms of dyspnea and exercise intolerance in any given individual. Moreover, CPET may identify the effects of skeletal muscle dysfunction differentiating between physical inactivity causes from impaired cardio-circulatory function. The absence of abnormal physiologic responses to CPET in a symptomatic subject requires careful evaluation (10). Thus, the aim of this study was to compare spirometry and CPET to detect ventilatory alterations in workers exposed to OEPM. The hypothesis is that CPET might be more efficient than spirometry to assess incipient ventilatory abnormalities.

\section{Material and Methods}

\section{Study design}

We conducted a cross-sectional study involving male workers. Participants were selected from the beginning of the epidemiology and human movement study (EPIMOV Study). Briefly, the EPIMOV Study is a population-based cohort study, which the main purpose is to investigate the associations of physical inactivity and sedentary behaviors with the occurrence of hypokinetic diseases. The Ethics and Research Committee on Human Beings of the Universidade Federal de São Paulo (UNIFESP) approved the study (\#186.796). All participants signed a consent form.

Demographic characteristics (age, gender, socioeconomic status, and educational level), self-reported medical history, anthropometrics, respiratory questionnaire (11), cardiovascular risk, spirometry, CPET, body composition (bioelectrical impedance), and level of physical activity in daily life (PADL) were collected from participants.

We included male workers older than 18 years with normal pulmonary function $(n=153)$ and excluded those who had musculoskeletal problems and lung and heart diseases previously diagnosed or identified during evaluation, previous thoracic surgery, previous history of mechanical ventilation, were using assistive gait devices, or had others problems identified in the electrocardiogram and issues that the researchers judged impeding for performing physical exercises safely.

Our exposure group (EG) and control group (CG) were defined according to a validated occupational respiratory questionnaire (i.e., ATS-DLD78), which was applied before the spirometric test. Those who answered "yes" to OEPM were included in the EG and who answered "no" were included in the CG.

The EG group was composed of individuals who reported exposure to many types of particulate matter during work, especially from port activities in Santos, SP, Brazil.

\section{Anthropometry}

Body mass to the nearest $0.1 \mathrm{~kg}$ and height to the nearest $0.1 \mathrm{~cm}$ were measured by a digital scale with a stadiometer $\left(\right.$ Toledo $^{\circledR}$, Brazil) according to standard recommendations previously described (12). The body mass index was then calculated $\left(\mathrm{kg} / \mathrm{m}^{2}\right)$.

\section{Respiratory assessment}

We applied a respiratory questionnaire based on a previously validated questionnaire developed by the American Thoracic Society (ATS) (11) to investigate pulmonary impairments and OEPM, which comprised: "Have you had any lung disease?", "Do you have or have had asthma?", "Have you ever underwent any thoracic surgery?", "Have you had to breathe through invasive mechanical ventilation?", "Have you ever worked in environments with high dust, smoke or other suspended chemicals for at least 1 year?"

Spirometry measurements were performed using a periodically calibrated spirometer (Quark PFT, Italy), according to the criteria established by the ATS (6). Forced vital capacity (FVC), forced expiratory volume in $1 \mathrm{~s}\left(\mathrm{FEV}_{1}\right)$, and $F E V_{1} / F V C$ ratio were analyzed. Obstructive lung disease was identified by $\mathrm{FEV}_{1} / \mathrm{FVC}<0.7$. Spirometric restrictive patterns were considered for FVC values $<80 \%$ of the predicted values (13) with $\mathrm{FEV}_{1} / \mathrm{FVC}>0.7$.

\section{Cardiopulmonary exercise testing}

CPET was performed on a treadmill ramp protocol (ATL, Ibramed, Brazil) in which the speed and inclination increase were individualized according to the predicted peak oxygen uptake (peak $\dot{\mathrm{V}}_{2}$ ). We designed CPETs to be completed in $10 \mathrm{~min}$ on average (range 8-12 $\mathrm{min}$ ). Metabolic, cardiovascular, and ventilatory responses were measured breath by breath through a gas analyzer (Quark PFT, Cosmed, Italy). The necessary air, gas mixture, and syringe calibrations were performed according to the manufacturer's recommendations. Pulmonary oxygen uptake $\left(\dot{\mathrm{V}}_{2}\right)$, carbon dioxide production $\left(\dot{\mathrm{V}} \mathrm{CO}_{2}\right)$, and minute ventilation ( $\dot{\mathrm{V} E})$ were measured throughout CPET and data were filtered through an arithmetic average every $15 \mathrm{~s}$ for further analysis. Heart rate (HR) was monitored throughout the test through a 12-lead electrocardiography (C12x, Cosmed). The anaerobic threshold (AT) was estimated by the $v$-slope method. The breathing reserve was calculated by subtracting the peak $\dot{V} E$ of maximum voluntary ventilation (MVV) estimated by age and gender of the participants (14). Subsequently, the breathing reserve index (BRI) was calculated by the following equation [(peak $\dot{V} E / M V V) \times 100]$ and reported as percentage, i.e., the higher the value, the lower the breathing reserve. Maximal exercise was determined in case of at least one of the following physiological indices: peak $\mathrm{HR}>85 \%$ of predicted for age $\left(220\right.$ - age), peak $\dot{\mathrm{V}} \mathrm{CO}_{2} / \mathrm{V}_{2}>1.0$, or oxygen uptake plateau (i.e., $\dot{\mathrm{VO}}_{2}$ maximum). Exercise intolerance was considered when peak $\dot{\mathrm{VO}}_{2}$ was below $83 \%$ of the predicted values (15). 
Additionally, the following submaximal relationships were determined throughout CPET: $\Delta \mathrm{HR} / \Delta \dot{\mathrm{V}} \mathrm{O}_{2}, \Delta \dot{\mathrm{VE}} /$ $\Delta \dot{\mathrm{V}} \mathrm{CO}_{2}$, and $\Delta \mathrm{VT} / \Delta \mathrm{In} \dot{\mathrm{V}} \mathrm{E}$. These relationships were obtained by linear regressions as previously described (14) and represent cardiovascular and ventilatory efficiency, and breathing pattern throughout the exercise, respectively.

We applied the natural logarithm in $\Delta V T / \Delta I n \dot{V} E$ as a strategy to linearize the relationship between VT and $V E$ since the increase of tidal volume is not linear during the maximal incremental test in relation to ventilation. This strategy allowed us to calculate the slope of the relationship in a simple linear regression.

\section{Body composition}

Body composition was determined by bioelectrical impedance (310E Biodynamics, USA). The resistance and reactance were obtained according to the standards and protocols developed by Kyle et al. (16). The lean body mass (LBM) and fat mass were calculated using the group-specific equations for healthy individuals (17).

\section{Physical activity in daily life}

The triaxial accelerometer (ActiGraph, MTI, USA) $(17,18)$ was attached to the waist by an elastic band above the dominant hip to evaluate the amount and intensity of PADL. The evaluations were performed over the course of 7 days. The validation of the data was based on the use of the device for at least 4 valid days with at least $720 \mathrm{~min}$ of monitoring. The time spent in sedentary, light, moderate, vigorous, and very vigorous physical activity was recorded and the number of steps/day was measured. We considered the minimal recommendations of PADL of 30 min of moderate to vigorous physical activity for at least 5 days per week (19). Those who had not performed this level of PADL were considered physically inactive.

\section{Statistical analysis}

Statistical analysis was performed using SPSS software version 23 (SPSS Inc., USA), with a significance of $P<0.05$. The normality of the sample was verified using the Kolmogorov-Smirnov test. Categorical variables are reported as frequency and percentage and we subsequently applied the chi-square test to verify the differences between groups. The association between categorical variables and OEPM was evaluated through $2 \times 2$ tables. Odds ratios (OR) and $95 \%$ confidence intervals $(95 \% \mathrm{Cl})$ were calculated. Continuous variables are reported as means $\pm S D$, and median and interquartile range (25-75\%). The Student's $t$-test or Mann-Whitney test were applied in the continuous variables to determine if there were significant differences between groups.

Multiple linear regression models were developed considering the spirometric indices and responses to CPET as primary outcomes, and OEPM as the main predictor. After bivariate analyses, we adjusted the multivariate models by the main confounders: age, gender, height, LBM, sedentary physical activity level (\%), socioeconomic status, education level, hypertension, diabetes mellitus, dyslipidemia, obesity, and smoking. These variables have been identified as correlated to pulmonary function and were included to check their influence in the multivariate analysis.

The sample size calculation took into account the number of clinically relevant predictors. We considered a total correlation coefficient of $r=0.80$ and a coefficient of determination of $R^{2}=0.64$. With alpha error of 0.05 and beta error of 0.80 , we achieved a sample of 10 observations for each variable included in the model. Considering that the models were adjusted for 12 variables, the sample was sufficient to answer our research question.

\section{Results}

Fifty-two participants reported "yes" to OEPM ("Have you ever worked in environments with high dust, smoke or other suspended chemicals for at least 1 year?") and were allocated in EG. The remaining 83 subjects composed the CG.

EG presented older age, larger amount of lean body mass $(\mathrm{kg})$, and lower educational level (Table 1). There was a significant association between higher educational level and OEPM (OR=0.290: 95\% $\mathrm{Cl}=0.100-0.842)$. EG reported many types of occupations with exposure to a large amount of particulate matter, especially materials from Santos port activities.

Although the absolute values of FEV1 and FVC were significantly different, we did not find spirometric differences in $\mathrm{FEV}_{1}(\%)$ and FVC (\%) between groups (Table 2).

Regarding PADL, participants of the EG showed a higher weekly amount of sedentary ( $77 \pm 8$ vs $74 \pm 8 \%$ ) and very light physical activity ( $14 \pm 5$ vs $12 \pm 4 \%$ ).

The influence of moderate-to-vigorous physical activity was analyzed as a categorical variable instead of as a continuous variable (i.e., physical inactivity) in multivariate analysis. We found no differences between groups.

Ten participants $(19.2 \%)$ in the EG and $8(9.6 \%)$ in the CG presented peak $\dot{\mathrm{V}}_{2}$ indicating exercise intolerance $(P=0.09)$. $E G$ presented worse physiological responses to CPET. There were significant differences for peak $\dot{\mathrm{VO}}_{2}$, $\dot{V E}, \mathrm{VT}, \mathrm{BRI}$, and end-expiratory pressure of $\mathrm{O}_{2}$. Regarding submaximal responses, the $E G$ presented worse $\Delta \mathrm{HR} / \Delta \dot{\mathrm{V}} \mathrm{O}_{2}, \Delta \mathrm{VT} / \Delta \mathrm{InVE}$, and absolute values of AT. Moreover, the AT as percentage of predicted peak $\mathrm{V}_{2}$ tended to be lower in the EG (Table 3).

In the multiple regressions, exposure to OEPM was not identified as an independent predictor of spirometric indices. The differences found in absolute values of $\mathrm{FEV}_{1}$ and FVC were attributed to difference in age and height. Regarding CPET, OEPM was identified as an independent predictor for peak $\dot{\mathrm{V} E}, \mathrm{BRI}$, peak $\mathrm{VT}$, and $\Delta \mathrm{VT} / \Delta \mathrm{InV} \mathrm{V}$ (Table 4). Therefore, OEPM was not a determinant for other measures of CPET. 
Table 1. General characteristics of the participants according to occupational exposure to particulate matter.

\begin{tabular}{lcc}
\hline Variables & CG $(\mathrm{n}=83)$ & EG $(\mathrm{n}=52)$ \\
\hline Age $(\text { years })^{*}$ & $37 \pm 11$ & $41 \pm 12$ \\
Height $(\mathrm{m})^{*}$ & $1.74 \pm 0.07$ & $1.70 \pm 0.07$ \\
Weight $(\mathrm{kg})$ & $86.5 \pm 16.3$ & $81.6 \pm 13.4$ \\
Body mass Index $\left(\mathrm{kg} / \mathrm{m}^{2}\right)$ & $28 \pm 5$ & $28 \pm 4$ \\
Body composition & & \\
$\quad$ Body fat (\%) & $23 \pm 7$ & $24 \pm 5$ \\
Body fat (kg) & $21 \pm 10$ & $20 \pm 7$ \\
Lean body mass (\%) & $77(70-84)$ & $74(69-79)$ \\
Lean body mass (kg) & $61(58-64)$ \\
Socio-economic level & $64(57-74)$ & \\
Low class & & $25(48.1 \%)$ \\
Medium class & $19(22.9 \%)$ & $10(19.2 \%)$ \\
High class & $35(42.2 \%)$ & $12(23.1 \%)$ \\
Educational level (incomplete high school) & $11(21.2 \%)$ \\
Cardiovascular risk & $16(19.3 \%)$ & \\
Hypertension & $6(7.2 \%)$ & $8(15.4 \%)$ \\
Diabetes mellitus & & $4(7.7 \%)$ \\
Dyslipidemia & $6(7.2 \%)$ & $13(25.0 \%)$ \\
Obesity & $3(3.6 \%)$ & $13(25.0 \%)$ \\
Smoking & $13(15.7 \%)$ & $8(15.4 \%)$ \\
Physical inactivity & $23(27.7 \%)$ & $4(7.7 \%)$ \\
\hline
\end{tabular}

Categorical data are reported as frequency (\%). Variables with normal distribution are reported as means $\pm \mathrm{SD}$, with non-normal distribution as median (interquartile range). CG: control group (not exposed); EG: exposed group. ${ }^{*} \mathrm{P}<0.05$ intergroup comparison (chi-square test for categorical data and Student's $t$-test or Mann-Whitney test for continuous variables).

Table 2. Comparison of spirometry data among participants exposed and not exposed to occupational particulates.

\begin{tabular}{lcc}
\hline Variables & CG $(n=83)$ & EG $(n=52)$ \\
\hline FVC $(L)^{*}$ & $4.81 \pm 0.79$ & $4.49 \pm 0.78$ \\
FVC $(\%)$ & $97 \pm 11$ & $95 \pm 10$ \\
FEV $_{1}(L)$ & $3.86 \pm 0.68$ & $3.61 \pm 0.71$ \\
FEV $_{1}(\%)$ & $95 \pm 12$ & $93 \pm 12$ \\
FEV $_{1} /$ FVC & $0.81 \pm 0.05$ & $0.80 \pm 0.06$ \\
PEF (L/s) & $9.94 \pm 1.76$ & $9.40 \pm 1.96$ \\
\hline
\end{tabular}

Data are reported as means \pm SD. CG: control group (not exposed); EG: exposed group. FVC: forced vital capacity; $\mathrm{FEV}_{1}$ : forced expiratory volume in $1 \mathrm{~s}$; PEF: peak expiratory flow. ${ }^{*} \mathrm{P}<0.05$ intergroup comparison (Student's $t$-test or MannWhitney test).

\section{Discussion}

The main findings of the present study showed that male workers exposed to OEPM for at least one year exhibited ventilatory alterations during exercise, such as shallower $\Delta \mathrm{VT} / \Delta \mathrm{InV}$ E and peak $\dot{\mathrm{V}}$, even with normal pulmonary function at rest. CPET has proven to be useful in identifying these early ventilatory changes related to OEPM.
Both CG and EG presented normal exercise responses, with no significant differences in the diagnosis of exercise intolerance $(P=0.09)$. We found shallower $\Delta V T / \Delta I n \dot{V} E$ in the $E G$ and this was determined solely by OEPM $\left(R^{2}=\right.$ 0.074). Moreover, we observed that OEPM was associated with the reduction of $8.4 \mathrm{~L} / \mathrm{min}$ in peak $\dot{\mathrm{V}}$ and $0.25 \mathrm{~L}$ in peak VT in CPET. Furthermore, the EG presented increased breathing reserve (i.e., lower BRI) at the end of CPET. These results clearly show that CPET was able to identify subtle ventilatory changes despite the absence of ventilatory limitations at rest.

CPET, as previously described in the literature, has been used to assess ventilatory impairments and exercise tolerance in many types of pneumoconiosis, such as in mine workers (20), asbestos workers (21), and people with silicosis (22). However, those studies did not focus in early detection in asymptomatic subjects as our study. The most important result of the present study was the shallower $\Delta V T / \Delta I n \dot{V} E$ ratio in the $E G$, which suggests tachypneic breathing pattern accompanied with less ventilatory efficiency during exercise.

Sperandio et al. (23) observed worse $\Delta \mathrm{VT} / \Delta \mathrm{In} \dot{\mathrm{V} E}$, lower peak $\dot{\mathrm{V}} \mathrm{O}_{2}, \dot{\mathrm{V}} \mathrm{E}$, and $\mathrm{VT}$ in adolescents with idiopathic scoliosis with the incremental shuttle walk test when compared to control group. Reduction on the compliance of 
Table 3. Maximal and submaximal physiological responses obtained during cardiopulmonary exercise test in stratified participants according to occupational exposure to particulate matter.

\begin{tabular}{|c|c|c|}
\hline Variables & $C G(n=83)$ & $E G(n=52)$ \\
\hline Test duration (min) & $9.34 \pm 1.28$ & $9.15 \pm 1.45$ \\
\hline \multicolumn{3}{|l|}{ Maximum metabolic response } \\
\hline$\dot{\mathrm{V}} \mathrm{O}_{2}(\mathrm{~mL} / \mathrm{min})^{*}$ & $3197(2666-3631)$ & $2816(2159-3454)$ \\
\hline$\dot{\mathrm{V}} \mathrm{O}_{2}\left(\mathrm{~mL} \cdot \mathrm{kg}^{-1} \cdot \mathrm{min}^{-1}\right)^{*}$ & $38.2 \pm 9.3$ & $35.4 \pm 9.6$ \\
\hline$\dot{\mathrm{VO}}_{2}(\% \text { pred })^{\star}$ & $101 \pm 15$ & $95 \pm 17$ \\
\hline Metabolic equivalent & $11 \pm 3$ & $10 \pm 3$ \\
\hline$\dot{\mathrm{V}} \mathrm{CO}_{2} / \mathrm{NO}_{2}{ }^{*}$ & $1.22 \pm 0.10$ & $1.17 \pm 0.10$ \\
\hline $\mathrm{PetCO}_{2}(\mathrm{mmHg})$ & $42.8 \pm 3.5$ & $42.9 \pm 4.5$ \\
\hline $\mathrm{PetO}_{2}(\mathrm{mmHg})^{*}$ & $111 \pm 3$ & $109 \pm 4$ \\
\hline \multicolumn{3}{|l|}{ Maximum ventilatory response } \\
\hline$\dot{\mathrm{V}} \mathrm{E}(\mathrm{L} / \mathrm{min})^{*}$ & $107 \pm 21$ & $91 \pm 25$ \\
\hline $\mathrm{BRI}(\%)^{*}$ & $67 \pm 14$ & $61 \pm 13$ \\
\hline $\mathrm{VT}(\mathrm{L})^{*}$ & $2.58 \pm 0.48$ & $2.26 \pm 0.42$ \\
\hline$f(r p m)$ & $41 \pm 7$ & $40 \pm 7$ \\
\hline$\dot{\mathrm{VE}} / \mathrm{NO}_{2}$ & $33.6 \pm 3.9$ & $32.3 \pm 4.8$ \\
\hline$\dot{\mathrm{VE}} / \mathrm{VCO}_{2}$ & $27.9 \pm 3.1$ & $28.3 \pm 4.1$ \\
\hline \multicolumn{3}{|l|}{ Maximum cardiovascular response } \\
\hline HR (\%pred.) & $94 \pm 8$ & $95 \pm 7$ \\
\hline$\dot{\mathrm{V}} \mathrm{O}_{2} / \mathrm{HR}\left(\mathrm{mL} \cdot \mathrm{min}^{-1} \cdot \mathrm{bpm}^{-1}\right)$ & $18.5 \pm 3.9$ & $17.0 \pm 4.7$ \\
\hline Systolic blood pressure $(\mathrm{mmHg})$ & $185(170-200)$ & $180(170-190)$ \\
\hline \multicolumn{3}{|l|}{ Submaximal relationship } \\
\hline AT $(\mathrm{mL} / \mathrm{min})^{*}$ & $2058(1675-2498)$ & $1851(1463-2150)$ \\
\hline AT $\left(\%\right.$ of $\mathrm{VO}_{2}$ peak) & $66 \pm 12$ & $66 \pm 9$ \\
\hline AT $\left(\% \dot{\mathrm{V}} \mathrm{O}_{2}\right.$ pred. peak) & $66(52-78)$ & $59(52-73)$ \\
\hline$\Delta \mathrm{HR} / \Delta \dot{\mathrm{VO}}_{2}\left(\mathrm{bpm} \cdot \mathrm{L}^{-1} \cdot \mathrm{min}^{-1}\right)$ & $35.5 \pm 9.0$ & $38.0 \pm 9.2$ \\
\hline$\Delta \dot{\mathrm{VE}} / \Delta \dot{\mathrm{V}} \mathrm{CO}_{2}$ & $24.4 \pm 3.1$ & $24.5 \pm 3.6$ \\
\hline$\Delta \mathrm{VT} / \Delta \mathrm{InV} \mathrm{E}^{*}$ & $0.94 \pm 0.27$ & $0.82 \pm 0.18$ \\
\hline
\end{tabular}

Data are reported as means $\pm S D$ or median and interquartile range. CG: control group (not exposed); EG: exposed group; $\dot{\mathrm{V}}$ : minute ventilation; $\dot{\mathrm{VO}}_{2}$ : oxygen uptake; $\dot{\mathrm{V}} \mathrm{CO}_{2}$ : carbon dioxide production; $\mathrm{BRI}$ : breathing reserve index; VT: tidal volume; f: respiratory rate; $\mathrm{HR}$ : heart rate; $\mathrm{PetCO}_{2}$ : end-expiratory pressure of $\mathrm{CO}_{2}$; PetoO ${ }_{2}$ : end-expiratory pressure of $\mathrm{O}_{2}$; AT: anaerobic threshold, $\Delta \mathrm{HR} / \Delta \mathrm{VO}_{2}$ : cardiac efficiency; $\Delta \dot{\mathrm{VE}} / \Delta \dot{\mathrm{V}}_{2}$ : ventilatory efficiency; $\Delta \mathrm{VT} / \Delta \mathrm{In} \dot{\mathrm{V} E}$ : breathing pattern. ${ }^{*} \mathrm{P}<0.05$ intergroup comparison (Student's $t$-test or Mann-Whitney test).

Table 4. Significant associations between occupational exposure to particulate matter and responses obtained in the cardiopulmonary exercise testing.

\begin{tabular}{lcccc}
\hline \multirow{2}{*}{ Outcomes } & \multicolumn{3}{c}{ Occupational exposure to particulate matter } \\
\cline { 2 - 5 } & $\mathrm{B}(\mathrm{SE})$ & $\mathrm{P}$ & $\Delta \mathrm{R}^{2}$ & Total $\mathrm{R}^{2}$ \\
\hline VंE peak (L/min) & $-8.451(0.162)$ & 0.028 & 0.043 & 0.407 \\
BRI (\%) & $-7.880(3.009)$ & 0.010 & 0.063 & 0.114 \\
VT peak (L) & $-0.251(0.088)$ & 0.006 & 0.068 & 0.192 \\
$\Delta \mathrm{VT} / \Delta \operatorname{InV} E$ & $-0.139(0.051)$ & 0.007 & 0.074 & 0.074 \\
\hline
\end{tabular}

Linear multiple regression models adjusted for age, gender, height, lean body mass, sedentary physical activity, socioeconomic status, educational level, hypertension, diabetes mellitus, dyslipidemia, obesity, and smoking. VE: minute ventilation; $\mathrm{BRI}$ : breathing reserve index; $\mathrm{VT}$ : tidal volume; $\Delta \mathrm{VT} / \Delta \mathrm{InV \dot {E }}$ : breathing pattern; SE: standard error. 
lung and/or chest wall cause changes in lung volumes and tachypnea during exercise (24). Furthermore, alterations on breathing pattern such as lower VT, increased respiratory effort, and rapid and shallow breathing were reported in individuals with interstitial lung diseases (25) and in patients with cystic fibrosis (26). Although the EG of the present study did not present evidence of scoliosis and spirometric restrictive pattern at rest, those participants exhibited a breathing pattern similar to that of patients with pulmonary restriction due to the limitation on the chest wall or intrapulmonary diseases. We believe that the difference in breathing pattern found between groups during CPET might be due to the exposure to a particulate matter. Thus, our results suggest that, among the several variables obtained in CPET, the $\Delta \mathrm{VT} / \Delta \mathrm{InV} E$ might be the most appropriate to differentiate workers with incipient ventilatory changes.

Changes in $\Delta V T / \Delta I n \dot{V} E$ might be a consequence of increased dead space to VT ratio $(26,27)$. To attend the ventilatory demand during exercise, the ventilatory system uses combinations of respiratory rate and VT. At the initial phase, increasing ventilation occurs by increasing VT to close to $70-80 \%$ of inspiratory capacity. As exercise intensity progresses, ventilation continues to increase as respiratory rate increases. During exercise, there is also a similarity between ventilation and pulmonary perfusion, which reduces the volume of physiological dead space and, consequently, the ventilatory demand. In individuals with interstitial lung disease or reduction on the compliance of lung and/or chest wall, there is a decrease in pulmonary volumes and an increase in dead space, so the ventilatory strategy during exercise becomes less efficient, i.e., the increase in ventilation occurs predominantly due to the increase in respiratory rate (tachypneic pattern) (28). Thus, the worse ventilatory efficiency observed in workers with pneumoconiosis was justified by greater values of ventilatory equivalent of oxygen during exercise (i.e., VंE/ $\dot{\mathrm{V}} \mathrm{O}_{2}$ ), which might have resulted from increased $\dot{\mathrm{V}} \mathrm{E}(29)$. The shallower slope of $\Delta \mathrm{VT} / \Delta \mathrm{In} \dot{\mathrm{V} E}$ observed in the present study might be associated with lower VT, which leads to worse ventilatory efficiency in response to exercise.

Volpino et al. (30) evaluated pulmonary function and CPET performed on a cycle ergometer in workers exposed to urban pollution. Despite the different methodology from this study, the authors observed some similar results. In addition to reduced peak $\dot{\mathrm{VO}}_{2}$ and $\mathrm{AT}$, the exposed workers also showed significant worsening in ventilatory equivalent of $\mathrm{CO}_{2}\left(\dot{\mathrm{V}} / \mathrm{VCO}_{2}\right)$ at $\mathrm{AT}$ and in the breathing reserve. These results indicate worse cardiorespiratory fitness associated with poor ventilatory efficiency in exposed participants. Although our study had a trend toward a higher proportion of participants with exercise intolerance, the results did not reach statistical significance. Volpino et al. (30) did not evaluate the breathing pattern as in the present study; we evaluated key submaximal relationships throughout CPET. The main advantage of the procedure proposed here is that submaximal relationships are not effort-dependent, i.e., even in low effort situations the early ventilatory changes described in the present study can be measured. Finally, $\Delta \mathrm{VT} / \Delta \mathrm{In} \dot{\mathrm{V} E}$ can be obtained using a simple ventilometry during ergometric tests, indicating the large potential of breathing pattern measurements to be routinely used for detecting early ventilatory abnormalities. This relationship clearly shows the breathing pattern during progressive exercise.

Differently from Volpino et al. (30), Schenker et al. (27) did not report a significant association between peak $\mathrm{VO}_{2}$ and OEPM. Inhalation of paraquat by harvest workers did not cause enough damage to lung function to result in exercise intolerance. However, they observed an association between the exposure to paraquat and worse VंE/ $\dot{\mathrm{V}} \mathrm{CO}_{2}$ at the end of the CPET performed on a cycle ergometer. These workers displayed oxygen desaturation during exercise, which is an indication of abnormalities in gas exchange, even in the absence of restrictive or interstitial lung impairments. Although peak $\dot{\mathrm{V} E} / \dot{\mathrm{V}} \mathrm{CO}_{2}$ is useful to identify ventilatory changes, the submaximal relations as presented in our study have the main advantage to perceive changes independent of maximum efforts, i.e., the $\Delta \mathrm{VT} / \Delta \mathrm{InV} \mathrm{V}$ can be evaluated even in individuals with low cardiorespiratory fitness as well as in subjects with poor effort during the CEPT.

Spirometry has been used by many studies to evaluate pulmonary function in subjects with respiratory occupational exposure $(4,31,32)$. However, the damages caused by OEPM do not always manifest in individuals at rest. Due to the increased metabolic demand under physical activity stress, impairments and alterations on the respiratory system become evident. Because altered spirometric indices are generally associated with alterations and disorders already installed, we believe that CPET is a useful tool for the early screening of ventilatory impairments in workers under OEPM.

Pulmonary function at rest was normal in both groups; nonetheless, CPET was able to identify discrete but significant changes in the breathing pattern during exercise. Discrepant results were reported by Bernardes et al. (33). Those authors found no difference in exposed and nonexposed lung function as measured by spirometry and functional capacity by the 6-minute walk test. According to the authors, the positive results were attributed to the use of personal protective equipment. We did not have information related to the use of appropriate protective equipment by our participants, which represent one of our limitations.

This study has some limitations. The EPIMOV study had the main purpose of diagnosing obstructive lung disease through the $\mathrm{FEV}_{1} / \mathrm{FVC}<70 \%$. For this reason, we did not perform diffusion capacity measurements and measurements of total lung capacity at rest. We were unable to assess changes of the relationship between dead space volume and VT. Blood gases analyses were 
not carried out in our epidemiological study, which would have substantially enriched our results. Another limitation is the self-reported OEPM. The workers reported exposure to a large range of materials related to inhalable particles found in port activities including truck traffic and transportation of grains and cereals. However, we do not have precise information regarding the type of gases nor the time of exposure. Finally, even with the air quality annually classified as moderate $\left(41-80 \mu \mathrm{g} / \mathrm{m}^{3}\right)$ to good $\left(0-40 \mu \mathrm{g} / \mathrm{m}^{3}\right)$, the urban pollution could have affected the subjects as well, since the port area of Santos has a high concentration of MP10 (34).

According to Ramos et al. (35), the level of physical activity influences the inhaled doses of air pollution due to increased inhalation rates. The devices we used to track physical activity level of our participants were unable to specify physical activity of labor time with no information regarding a labor schedule. Therefore, we did not use physical activity compared to intensity of the labor and of the inhalation of particulate material. Moreover, we assessed the influence of moderate-to-vigorous physical activity as a continuous variable instead of a dichotomous variable

\section{References}

1. Seaton A, MacNee W, Donaldson K, Godden D. Particulate air pollution and acute health effects. Lancet 1995; 345: 176-178, doi: 10.1016/S0140-6736(95)90173-6.

2. Sjögren B. Occupational exposure to dust: inflammation and ischaemic heart disease. Occup Environ Med 1997; 54: 466-469, doi: 10.1136/oem.54.7.466.

3. Suadicani P, Hein HO, Gyntelberg F. Airborne occupational exposure, $A B O$ phenotype and risk of ischaemic heart disease in the Copenhagen Male Study. J Cardiovasc Risk 2002; 9: 191-198, doi: 10.1097/00043798-200208000-00003.

4. Ulvestad B, Lund MB, Bakke B, Thomassen Y, Ellingsen DG. Short-term lung function decline in tunnel construction workers. Occup Environ Med 2014; 108-113, doi: 10.1136/ oemed-2014-102262.

5. Drummond MB, Hansel NN, Connett JE, Scanlon PD, Tashkin DP, Wise RA. Spirometric predictors of lung function decline and mortality in early chronic obstructive pulmonary disease participant characteristics at baseline. Am J Respir Crit Care Med 2012; 185: 1301-1306, doi: 10.1164/rccm. 201202-0223OC.

6. Miller MR, Hankinson J, Brusasco V, Burgos F, Casaburi R, Coates A, et al. Standardization of spirometry. Eur Respir J 2005; 26: 319-338, doi: 10.1183/09031936.05.00034805.

7. Universities Occupational Safety and Health Educational Resource Center. "NIOSH spirometry training guide". http:// www.cdc.gov/niosh/docs/2004-154c/pdfs/2004-154c.pdf. Accessed Nov 11, 2015.

8. Balady GJ, Arena R, Sietsema K, Myers J, Coke L, Fletcher GF, et al. Clinician's guide to cardiopulmonary exercise testing in adults: A scientific statement from the American heart association. AHA Circulation 2010; 122: 191-225, doi: 10.1161/CIR.0b013e3181e52e69. for physical inactivity in the multivariate analysis, and found no differences in the results.

Despite these limitations, our results indicated excellent validity since after adjusting for confounder variables in the multivariate regression model, OEPM remained an independent predictor of the worst breathing pattern. Therefore, one simple question could track subjects with subclinical ventilatory changes. The results presented here should be interpreted with caution for women, given the gender differences in physiological responses to CPET (15).

We concluded that CPET is a promising tool for screening workers under OEPM, with the capacity to identify incipient ventilatory changes. CPET might be a more sensitive feature for assessing early pulmonary impairment related to OEPM, useful for the prevention of occupational respiratory diseases in asymptomatic workers.

\section{Acknowledgments}

This work was supported by Fundação de Amparo à Pesquisa do Estado de São Paulo (FAPESP; \#2011/ 07282-6).
9. Dwyer GB, Davis SE, American College of Sports Medicine. Guidelines for exercise testing and prescription. Dwyer GB, Davis SE (Associate editors) Pire NI WRT (Editor). ACSM's health-related physical fitness assessment manual. 2nd edn. Philadelphia: Wolters Kluwer/Lippincott Williams \& Wilkins, c2008.; 2008. p 191.

10. Elbehairy AF, Parraga G, Webb KA, Neder JA, O'Donnell $D E$. Mild chronic obstructive pulmonary disease: why spirometry is not sufficient! Canadian Respiratory Research Network (CRRN). Expert Rev Respir Med 2017; 11: 549-563, doi: 10.1080/17476348.2017.1334553.

11. ATS II. Recommended respiratory disease questionnaire for use with adults and children in epidemiological research. Am J Respir Crit Care Med 1978; 118: 7-53.

12. National Health and Nutrition, Examination Survey (NHANES). "National Youth Fitness Survey (NYFS) body measures procedures manual" http://www.cdc.gov/nchs/data/nnyfs/Body_ Measures.pdf. Accessed Nov 11, 2015.

13. Pereira CAdeC, Sato T, Rodrigues SC. Novos valores de referência para espirometria forçada em brasileiros adultos de raça branca. $J$ Bras Pneumol 2007; 33: 397-406, doi: 10.1590/S1806-37132007000400008.

14. Neder JA, Fernandes ANG, Silva AC, Cabral ALB, Nery LE. Relationship between aerobic fitness and clinical indicators of asthma severity in children. Braz J Pneumol 1998; 24: 3-10.

15. Wasserman K, Hansen JE, Sue DY, Stringer SWW, Sietsema KE, Sun XG, et al. Principles of exercise testing and interpretation: including pathophysiology and clinical applications. 5th edn. Philadelphia, Lippincott Williams \& Wilkins; 2012. p 2166

16. Kyle UG, Bosaeus I, De Lorenzo AD, Deurenberg P, Elia M, Gómez JM, et al. Bioelectrical impedance 
analysis - part I: review of principles and methods. Clin Nutr 2004; 23: 1226-1243, doi: 10.1016/j.clnu.2004. 06.004.

17. Kyle UG, Genton L, Karsegard L, Slosman DO, Pichard C. Single prediction equation for bioelectrical impedance analysis in adults aged 20-94 years. Nutrition 2001; 17 : 248-253, doi: 10.1016/S0899-9007(00)00553-0.

18. Troiando RP, Berrigan D, Dodd KW, Mâsse LC, Tilert Ti, Mcdoweell M. Physical activity in the United States measured by accelerometer. Med Sci Sports Exerc 2008; 40: 181-188, doi: 10.1249/mss.0b013e31815a51b3.

19. AHA. "American Heart Association recommendations for physical activity in adults".http://www.heart.org/HEARTORG/ GettingHealthy/PhysicalActivity/FitnessBasics/AmericanHeart-Association-Recommendations-for-Physical-Activity-inAdults_UCM_307976_Article.jsp\#.VkVGV66rTVo. Accessed Nov 11, 2015.

20. Petsonk EL, Rose C, Cohen R. Coal mine dust lung disease. New lessons from old exposure. Am J Respir Crit Care Med 2013; 187: 1178-1185, doi: 10.1164/rccm.201301-0042Cl.

21. Miller A, Anand B, Sloane MF, Brown LK, Teirstein AS. Cardiorespiratory responses to incremental exercise in patients with abestos-related pleural thickening and normal or slightly abnormal lung function. Chest $J$ 1993; 1045-1050, doi: 10.1378/ chest.103.4.1045.

22. Violante B, Brusasco V, Buccheri G. Exercise testing in radiologically-limited, simple pulmonary silicosis. Chest 1986; 90: 411-415, doi: 10.1378/chest.90.3.411.

23. Sperandio EF, Alexandre AS, Yi LC, Poletto PR, Gotfryd AO, Vidotto MC, et al. Functional aerobic exercise capacity limitation in adolescent idiopathic scoliosis. Spine J 2014; 14: 2366-2372, doi: 10.1016/j.spinee.2014.01.041.

24. Harty HR, Corfield DR, Schwartzstein RM, Adams L. External thoracic restriction, respiratory sensation, and ventilation during exercise in men. J Appl Physiol 1999; 86: 1142-1150, doi: 10.1152/jappl.1999.86.4.1142.

25. Harris-Eze AO, Sridhar G, Clemens RE, Zintel TA, Gallagher CG, Marciniuk DD. Role of hypoxemia and pulmonary mechanics in exercise limitation in interstitial lung disease. Am J Respir Crit Care Med 1996; 154: 994-1001, doi: 10.1164/ ajrccm.154.4.8887597.
26. Renzi G, Milic-Emili J, Grassino AE. The pattern of breathing in diffuse lung fibrosis. Bull Eur Physiopathol Respir 1982; 18: $461-472$.

27. Schenker MB, Stoecklin M, Lee K, Lupercio R, Zeballos RJ, Enright $P$, et al. Pulmonary function and exercise-associated changes with chronic low-level paraquat exposure. Am J Respir Crit Care Med 2004; 170: 773-779, doi: 10.1164/ rccm.200403-266OC.

28. Cotes JE, Field GB. Lung gas exchange in simple pneumoconiosis of coal workers. Br J Ind Med 1972; 29: 268-273, doi: 10.1136/oem.29.3.268.

29. Bauer TT, Schultze-Werninghaus G, Kollmeier J, Weber A, Eibel R, Lemke B, et al. Functional variables associated with the clinical grade of dyspnoea in coal miners with pneumoconiosis and mild bronchial obstruction. Occup Environ Med 2001; 58: 794-799, doi: 10.1136/oem.58.12.794.

30. Volpino P, Tomei F, La Valle C, Tomao E, Rosati MV, Ciarrocca M, et al. Respiratory and cardiovascular function at rest and during exercise testing in a healthy working population: Effects of outdoor traffic air pollution. Occup Med 2004; 54: 475-482, doi: 10.1093/occmed/kqh102.

31. Myers J, Buchanan N, Walsh D, Kraemer M, McAuley P, Hamilton-Wessler $\mathrm{M}$, et al. Comparison of the ramp versus standard exercise protocols. J Am Coll Cardiol 1991; 17: 1334-1342, doi: 10.1016/S0735-1097(10)80144-5.

32. Bhambhani $Y$, Burnham R, Snydmiller G, MacLean I, Lovlin R. Effects of 10-ppm hydrogen sulfide inhalation on pulmonary function in healthy men and women. $J$ Occup Environ Med 1996; 38: 1012-1017, doi: 10.1097/00043764$199610000-00011$.

33. Bernardes RAB, Chiavegato LD, Moraes MV, Negreiros A, Padula RS. Lung function and functional capacity among foundry workers using effective risk control measures. J Work 2015; 1-8.

34. Environmental Protection Agency in São Paulo (CETESB). "Qualidade do Ar". http://sistemasinter.cetesb.sp.gov.br/Ar/ php/mapa_qualidade_rmsp.php. Accessed August 11, 2017.

35. Ramos CA, Reis JF, Almeida T, Alves F, Wolterbeek HT, Almeida SM. Estimating the inhaled dose of pollutants during indoor physical activity. Sci Total Environ 2015; 527528: 111-118, doi: 10.1016/j.scitotenv.2015.04.120. 